\title{
Study of Lead-Free Ferroelectric Composite Coatings by Impedance Spectroscopy
}

\author{
Mariya Aleksandrova ${ }^{1, *}$, Tsvetozar Tsanev ${ }^{1}$, Ivailo Pandiev ${ }^{2}$ and Georgi Dobrikov ${ }^{1}$ \\ 1 Department of Microelectronics, Technical University of Sofia, Sofia, Bulgaria \\ 2 Department of Electronics, Technical University of Sofia, Sofia, Bulgaria \\ * Correspondence: m_aleksandrova@tu-sofia.bg
}

Received: 29 February 2020; Accepted: 6 May 2020; Published: 7 May 2020

\begin{abstract}
The aim of the study is preparation and electrical characterization of lead-free ferroelectric oxide $\mathrm{BaSrTiO}_{3}$ in composition with piezoelectric polymer. The properties of the deposited films were compared with pristine oxide. Atomic force microscopy showed smooth surface, regular and homogeneous distribution of particles of both components in the composite films. The dielectric properties (electric permittivity and dielectric loss) were investigated at different temperatures ranged from $5{ }^{\circ} \mathrm{C}$ to $130{ }^{\circ} \mathrm{C}$. Impedance spectroscopy was applied in the frequency range $100 \mathrm{~Hz}-$ $100 \mathrm{kHz}$. It was demonstrated that the dielectric constant increase with addition of piezoelectric polymer to the ceramic phase. It can be seen that the interface conditions at the electrodes are improved after inserting piezoelectric polymer. Interpretation of the plots of the complex impedance vs. frequency and real part of the impedance vs. imaginary part, gives information about the polarization process, revealing in the structures.
\end{abstract}

Keywords: ferroelectric composite; piezoelectric polymer; impedance spectroscopy; dielectric properties; polarization processes

\section{Introduction}

Recently, the microstructure and dielectric properties of the barium titanate based materials have been widely studied because of their importance in the lead-free feroelectric sensor technology [1]. Variety derivatives have been studied and different approaches, such as doping and nanostructuring have been applied to gain mostly the piezoelectric response in $\mathrm{BaTiO}_{3}$ and $\mathrm{SrTiO}_{3}$ based applications. The studies have revealed that the ferroelectric properties can be controlled by the deposition methods and the deposition conditions. To establish a relation between the microstructure and the ferroelectric response, a scanning electron microscopy (SEM), or atomic force microscopy (AFM) have been applied in combination with polarization P-E hysteresis curves [2]. However, information about some fundamental processes, such as dipoles motion, which are responsible for the polarization processes, cannot be directly accessed from the SEM/AFM and P-E measurements. Therefore, more sophisticated technique is necessary, such as impedance spectroscopy (IS). It relies on bias signal supplied to the sample, which can vary in magnitude and frequency [3]. Parameters like the full impedance and admittance, contact resistance, interface capacitance, dielectric permittivity and loss tangent can be extracted from the impedance measurements. In addition, some major parameters of the ferroelectric structures, extracted from the IS can be determined at different temperatures. All these parameters are important for the sensor technology, because they are related to linearity of the response (contact resistance), time delay of the response (interface capacitances and their frequency dependence), thermal stability (dielectric permittivity and losses vs temperature), impedance matching with the sensor signal's processing circuit (impedance/admittance of the sample), etc. It was found that the ferroelectric ceramic based 
structures, which are not fine granular has exhibited faster degradation of their electrical characteristics, which has been ascribed to the worsen contact properties between the grains (which are very often sharper depending on the materials' nature and crystallization degree) and the electrode films. Therefore, a buffer layer for interface smoothening is welcome. The poly(vinylidene fluoride-co-trifluoroethylene) fluoropolymer resin (PVDF-TrFE) is suitable candidate for this purpose. In our previous study, we have found that printed PVDF-TrFE on $\mathrm{BaSrTiO}_{3}$ (BST) sputtered film exhibited an excellent performance, as well as that a composition between them has arose due to diffusion of the PVDF-TrFE particles between the crystallites of the BST [4]. This combination has been explored for energy harvesting and energy storage applications [5]. However, the effect of the composition PVDF-TrFE/BST on the ferroelectric sensors' contact resistance, interface capacitance, full impedance and their frequency and temperature dependences has not been investigated yet. By the authors' knowledge this is the first time application of the impedance spectroscopy for investigation of ferroelectric sensors on silicon with lead-free ceramic/polymer composite functional films.

\section{Methods}

Silicon wafers with orientation (100) were used as substrates. They were preliminary cleaned from the native $\mathrm{SiO}_{2}$ by rinsing in $10 \%$ water solution of hydrofluoric acid, followed by sonication bath in acetone. $\mathrm{BaSrTiO}_{3}$ was $\mathrm{RF}$ sputtered on silver coated silicon wafers without additional oxidation of the target and without post-annealing of the films. The sputtering pressure was set to 2.5.10-2 Torr and the sputtering voltage was $0.75 \mathrm{kV}$, defining plasma power of $43 \mathrm{~W} /$ inch. The bottom and top electrodes were made of thermally evaporated silver films due to the great thermal and electrical conductivity of the silver. The top electrodes were patterned by lift-off process to produce variety of similar segments for impedance spectroscopy probing. The ferroelectric ink PVDF-TrFE was spin coated at $1000 \mathrm{rpm}$ from Solvene 300 solution and then annealed at $120^{\circ} \mathrm{C}$ in oxygen atmosphere for $15 \mathrm{~min}$ to gain its ferroelectric phase. Atomic force microscopy was conducted by using AFM model MFP-3D, Asylum Research, Oxford Instruments in non-contact mode for 2D and 3D imaging of the PVDF-TrFE coated and uncoated BST films' surfaces. Impedance spectroscopy was carried out by a chemical impedance analyzer IM3590 Hioki in the frequency range in the frequency range $100 \mathrm{~Hz}-100 \mathrm{kHz}$. The temperature measurements in the range $5{ }^{\circ} \mathrm{C}-130{ }^{\circ} \mathrm{C}$ were realized by a home-made Peltier based heating-cooling system with smooth regulation of the temperature.

\section{Results and Discussion}

Figure 1 shows compared 2D and 3D AFM images of $\mathrm{BaSrTiO}_{3}$ (left half of the images) and PVDF-TrFE coated BST (right half of the images). They reveal that large agglomerates in the range of 10 micrometer grains of perovskite phase are formed at BST sputtering on the silicon. On the top view it seems that a dense microstructure is formed, however, the tilted 3D image shows that there is a great variation in the BST film height at the interface area between the large crystallites (the height difference between the largest hill and hole was approximately $211 \mathrm{~nm}$ related to a total thickness of $480 \mathrm{~nm}$ ). The spin-coating of the polymeric solution caused gaps filling and smoothening of the BST film, as can be clearly seen from both images. Finer microstructure with an average roughness of less than $100 \mathrm{~nm}$ was observed for the smoother surface. It is expected that the improved film's flatness will result in a decrease of the losses and contact resistance due to the increased contact area at the interfaces electrode/functional film. 

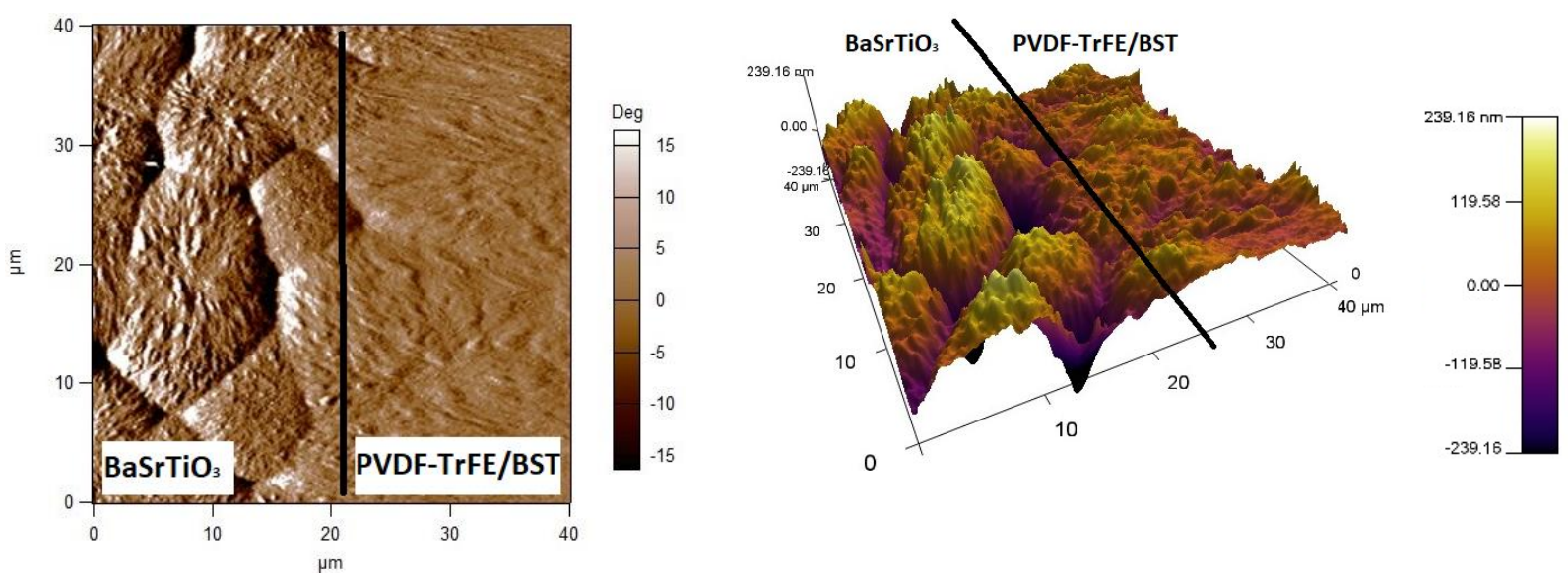

Figure 1. 2D and 3D AFM images comparing the surface topography of BST film and PVDF-TrFE coated BST film.

Dielectric constant, $\varepsilon_{\mathrm{r}}$, and loss factor, $\mathrm{D}$, were measured within the temperature range from $5^{\circ} \mathrm{C}$ to $130{ }^{\circ} \mathrm{C}$ (further temperature increase would change the crystal phase of the PVDF-TrFE polymer) at frequencies $100 \mathrm{~Hz}, 1 \mathrm{kHz}, 10 \mathrm{kHz}$, and $100 \mathrm{kHz}$, for pristine BST sample and PVDF-TrFE/BST. It was found that the dielectric permittivity is greater for the composite PVDF-TrFE/BST that can be ascribed to interfacial polarization at the ceramic/polymer interface due to the difference of their conductivity and piezoelectric coefficients. It was also found that $\varepsilon$ r slightly decreased with the temperature for all set frequencies (Figure 2). Dielectric losses were found to be smaller for the PVDFTrFE/BST, slightly dependent on the temperature and more strongly dependent on the frequency (Figure 3). The results are in good agreement with the reported for ferroelectric composites [6].

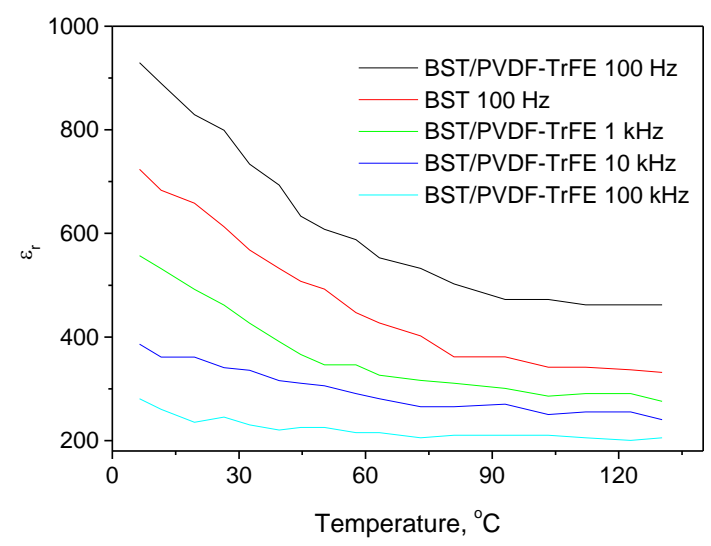

Figure 2. Variation of the permittivity with the temperature, frequency and composition of the functional film. 


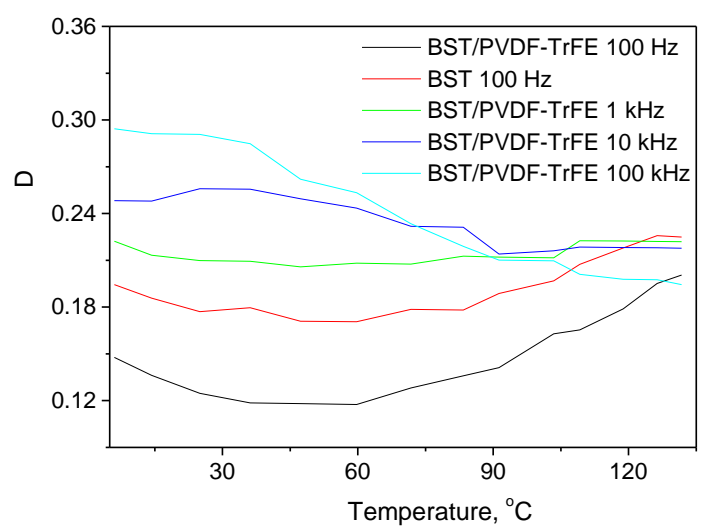

Figure 3. Variation of the loss factor with the temperature, frequency and composition of the functional film.

\section{Conclusion}

AFM images show smooth surface after insertion of PVDF-TrFE coating onto the BST surface. Uniformly distributed peaks form the average roughness of the film. The structure with composite film show high value of $\varepsilon_{r}$ and low loss factor, with poor temperature dependence, which is favorable for sensing applications relying on capacitor, or piezoelectric principle. Future work will be related to full impedance measurements at different frequencies, analysis of the real and imaginary part behavior and relation with the polarization processes in the ferroelectric film.

Acknowledgments: This study was funded by the BNSF, grant number KP06-H27/1. The authors are thankful to the colleagues from the Bulgarian Academy of Sciences and from the Govt. V.Y.T Autonomous College, Durg, India for the microscopic imaging of the samples.

\section{References}

1. J. A. Krishnaswamy et.al., Smart Materials and Structures, Vol. 29, No 1, 2019.

2. S. Zhou et. al., Materials Horizons, 7, 263-274, 2020.

3. T. Schenk, M. Hoffmann, M. Pešić, M. H. Park, C. Richter, U. Schroeder, and T. Mikolajick, Phys. Rev. Applied 10, 064004, 2018.

4. I Pandiev, M Aleksandrova, G Kolev, 5th International Conference on Energy Engineering and Smart Materials ICEESM, April 15 - 17, 2020, Barcelona, Spain.

5. Shihui Yu, Chunmei Zhang, Muying Wu, Helei Dong, Lingxia Li, Journal of Power Sources, 412, 648-654, 2019.

6. C. R. Gautam, D. Kumar, Prabhakar Singh, and O. Parkash, International Scholarly Research Network ISRN Spectroscopy, Volume 2012, Article ID 410583, 2012.

(C) 2020 by the authors. Submitted for possible open access publication under the terms and conditions of the Creative Commons Attribution (CC BY) license (http://creativecommons.org/licenses/by/4.0/). 